Gesundheit im Judentum: Ein interkultureller Diskurs über Unverständnis und Missverständnis in Vergangenheit und Gegenwart

In:

Philip van der Eijk / Detlev Ganten / Roman Marek (Hrsg.): Was ist Gesundheit? :

Interdisziplinäre Perspektiven aus Medizin, Geschichte und Kultur

ISBN: 978-3-11-071333-6. - Berlin / Boston: De Gruyter, 2021

(Humanprojekt : Interdisziplinäre Anthropologie ; 18)

Teil 2: Gesundheitsbegriffe regionaler Medizintraditionen

S. $97-108$ 


\title{
Gesundheit im Judentum: Ein interkultureller Diskurs über Unverständnis und Missverständnis in Vergangenheit und Gegenwart
}

\begin{abstract}
Health in Judaism: An Intercultural Discourse on Lack of Understanding and Misunderstanding in the Past and Present. Hardly any other religion pays as much attention to physical health as Judaism. Beginning with the Torah, the contrast between „healthy“ and „sick“ is already conceptualized and associated with the will of God and his plan of creation. In addition to the stereotype that Jews are sicker than their fellow human beings, there is an early claim that their state of health is better than that of other peoples. The religious writings of Judaism contain a large number of regulations that show how much the GrecoRoman doctrine of dietetics has been internalized, expanded and adapted to one's own spiritual needs. There is broad consensus among today's rabbis that health care, as described above all in the Talmud, was time-related and therefore should be based on today's standards and findings while remaining in compliance with religious laws.
\end{abstract}

\section{Die besondere Wertschätzung der Gesundheit im Judentum}

„Ein bisschen Sonne, ein bisschen Regen, ein ruhiger Ort sich hinzulegen, Hauptsache man kann glücklich sein. Ein Paar Schuhe, ein Paar Socken, ein Kleid ohne Flicken, ein Täschchen mit drei, vier Geldstücken. Hauptsache gesund, dann kann man glücklich sein. Die Luft kostet nichts“, so lautet die deutsche Übersetzung eines bekannten jiddischen Liedes. Darin wird zum Ausdruck gebracht, dass Gesundheit wichtiger ist als irdische Reichtümer.

Anmerkung: Der vorliegende Artikel basiert im Kern auf meiner Monographie Leib und Leben im Judentum (Jütte 2016), wurde zum Teil aber noch ergänzt und aktualisiert.

Robert Jütte, Institut für Geschichte der Medizin der Robert Bosch Stiftung (IGM), Stuttgart

Ә OpenAccess. (C 2021 Robert Jütte, publiziert von De Gruyter. (cc))BY-NC-SA Dieses Werk ist lizenziert unter einer Creative Commons Namensnennung - Nicht kommerziell - Weitergabe unter gleichen Bedingungen 4.0 International Lizenz. https://doi.org/10.1515/9783110713336-009 
Kaum eine andere Religion schenkt der physischen Gesundheit so viel Beachtung wie das Judentum. Man denke nur an die berühmten Sentenzen im Buch Jesus (Ben) Sirach:

Es ist besser, arm zu sein und dabei frisch und gesund als reich und nicht gesund. Gesund und frisch sein ist besser als alles Gold, und ein gesunder Körper ist besser als großes Gut. Kein Reichtum ist zu vergleichen mit einem gesunden Körper, und kein Gut gleicht der Freude des Herzens (Sirach 30:14-16).

Bereits in der Thora wird der Gegensatz zwischen „gesund“ und „krank“ konstruiert und mit dem Willen Gottes und seinem Schöpfungsplan in Verbindung gesetzt. Ähnlich bringt es das traditionelle Gebet eines jüdischen Arztes auf den Punkt, das lange Zeit fälschlicherweise dem berühmten jüdischen Philosophen und Mediziner Maimonides zugeschrieben wurde: „Allgütiger! Du hast des Menschen Leib voller Weisheit gebildet. Zehntausendmal zehntausend Werkzeuge hast du in ihm vereint, die unabläßig thätig sind, um das schöne Ganze, die Hülle der Unsterblichen, zu erhalten und zu ernähren“ (Leder 2007, S. 238). Der menschliche Körper ist also ein Wunderwerk Gottes, wie es zu Anfang dieses Gebetes heißt; dennoch ist der Mensch nicht vor Krankheiten gefeit.

Das antike Judentum teilte das Gesundheitsverständnis, das in seinen Grundzügen metaphysisch ist, mit seiner Umwelt, den vorderasiatischen Hochkulturen (Kaiser 2002, S. 9). Der einzige Unterschied ist, dass es im Judentum keine Vielzahl an Göttern, sondern nur einen Gott gibt, der die Menschheit wegen Ungehorsams mit einem Verlust an Gesundheit strafen kann. Krankheit wird also als Folge des unergründlichen Zorns Gottes oder als Strafe Jahwes angesehen, wie es in den Psalmen 6 und 88 zum Ausdruck kommt. Wer gesund bleiben will, tut also gut daran, Gottes Gebote einzuhalten (2. Mose 15:26; 23:25). Vor allem in nachexilischer Zeit entstand die Vorstellung, dass sich Gott dabei bestimmter „Werkzeuge“ bedient. An erster Stelle ist hier an den Satan oder an Krankheitsdämonen zu denken. Zur Wiederherstellung der Gesundheit griff Gott dagegen auf Engel zurück (vgl. Tobias 12:5-20). Der Arzt, bereits in der Bibel gelegentlich erwähnt, steht nicht in Konkurrenz zur göttlichen Heilkunst, er ist ebenfalls ein Werkzeug Gottes: „Befreunde dich mit dem Arzt, ehe du ihn brauchst, / denn auch ihm hat Gott (seine Aufgabe) zugeteilt. / Von Gott stammt der Sachverstand des Arztes, und vom König erhält er seine Bezüge“ (Sirach 38:1-2). 


\section{Gesünder oder kränker als andere Völker und Religionen}

Neben dem gängigen Stereotyp, dass Juden krankheitsbehafteter sind als ihre Mitmenschen, findet sich schon früh die Behauptung, dass ihr Gesundheitszustand besser sei als der anderer Völker (Levin \& Prince 2010, S. 567-568). Bereits Tacitus (um 58 - um 120), der kein Judenfreund war, hob die robuste Gesundheit der Juden im damaligen Palästina hervor: „Die Menschen sind körperlich gesund und stark im Ertragen von Strapazen“ (Historien, VI.1). Der evangelische Theologe Johannes Buxtorf d. Ä. behauptete in seinem Werk Synagoga Judaica, ohne dies allerdings im Einzelnen zu belegen oder näher zu begründen, dass Juden länger lebten als Christen und im Unterschied zu den zuletzt Genannten auch immun gegen alle möglichen Krankheiten seien (Buxtorf 1643, S. 620).

Im 19. Jahrhundert nahmen sich jüdische und christliche Ärzte dieses Themas an und bezeichneten die mit dem jüdischen Ritus verbundenen Hygienevorschriften als gesundheitsfördernd (Jütte 2016, S. 239-261; Wolff 2014, S. 166 235). Rituelle Waschungen bei den verschiedensten Anlässen (vor dem Morgengebet und Synagogenbesuch, nach dem Stuhlgang etc.), Reinigungsbäder nach der Menstruation sowie andere religiöse Vorschriften mit langer Tradition, die sich medizinisch deuten ließen, wurden beispielsweise auf der Dresdener Hygiene-Ausstellung von 1911 und der Gesolei-Ausstellung in den 1920er Jahren im Sinne einer modernen und fortschrittlichen Hygiene interpretiert oder umgedeutet (Baneth 1912, bes. S. 83 ff.; Wiesemann 1993).

Das Gleiche lässt sich für die Sexualmoral und die Speisegesetze feststellen. Aus der Vielzahl der zeitgenössischen Stimmen, die kurz vor oder nach der Wende vom 19. zum 20. Jahrhundert die spezifische Diätetik des Judentums als Krankheitsprophylaxe würdigten, war es kein Geringerer als Rudolf Virchow, der auf der 58. Versammlung der Naturforscher und Ärzte (1885) die Frage stellte,

inwieweit die sonderbare Immunität, welche die Juden unter den verschiedensten Umständen [...] gezeigt haben, basiert auf der Besonderheit ihres Lebens, auf der strengeren hygienischen Haltung des Hauses, auf der größeren Sorgfalt der Speisegesetze, auf dem mehr häuslichen Leben und dergleichen (zitiert in Grunwald 1912, S. 299).

Virchow ließ die Frage offen, doch machte er zumindest durch eine Nachbemerkung deutlich, dass er im Unterschied zu zahlreichen Zeitgenossen wenig von rassenbiologischen Erklärungen hielt, wenn er sie auch nicht gänzlich ausschließen wollte. Sein amerikanischer Kollege Ephraim M. Epstein, der in Wien und in Russland praktiziert hatte, bevor er sich in Cincinnati (Ohio) als Arzt 


\title{
Die Hygiene der Juden
}

\author{
Im Anschluß an die Internationale \\ Hygiene-Ausstellung Dresden 1911
}

herausgegeben

von

Dr. MAX GRUNWALD.

Verlag der Historischen Abteilung der Internationalen Hygiene-Ausstellung

::: Dresden 1911 :::

DRESDEN N., Großenhainerstraße 9

Abb. 1: Titelblatt Die Hygiene der Juden, Dresden 1911. (C) Institut für Geschichte der Medizin der Robert Bosch Stiftung 


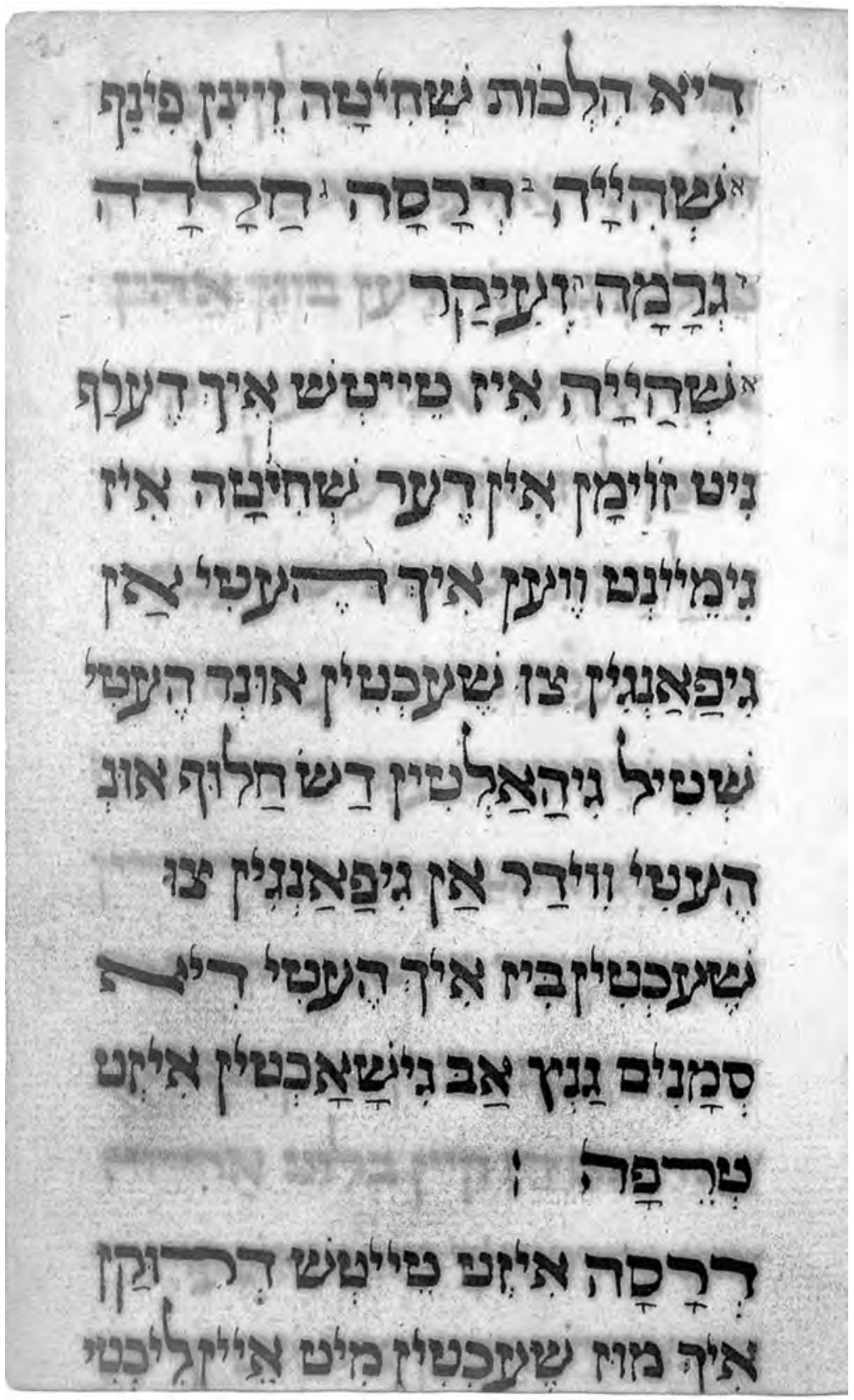

Abb. 2: Vorschriften für das Schächten (Jiddisch). @ Wellcome Collection. Attribution 4.0 International (CC BY 4.0) 
niederließ, akzeptierte dagegen den Hinweis auf religiöse Speisegesetze nicht als Begründung für die geringere Morbidität der Juden. Er machte stattdessen andere Faktoren (starke Familienbande und Solidarität sowie eine hochentwickelte Sozialfürsorge) für den meist besseren Gesundheitszustand der jüdischen Bevölkerung verantwortlich (Epstein 1874; Gilman 1995, S. 110). Fast zur gleichen Zeit vertrat ein amerikanischer Reformrabbiner dagegen die These, dass die vergleichsweise geringe Morbidität unter den Juden vor allem dem Umstand zu verdanken sei, dass diese ihre religiösen Reinheitsgebote streng einhielten (Krauskopf 1889, S. 7). Selbst in der neueren medizinischen Literatur findet sich ein Reflex dieser Diskussion um Judentum und Hygiene, wenn beispielsweise zur Erklärung niedriger Krebsraten (in diesem Falle Penis- bzw. Cervixkarzinom) unter Juden auf das religiöse Beschneidungsgebot verwiesen wird (Schuster 1980, S. 63-64; Susser \& Watson 1972, S. 75).

Die Diskussion um die gesundheitlichen Vorzüge, ein Jude zu sein, widerspricht der antisemitischen Vorstellung, die Krankheit und Judentum gleichsetzt, sowohl im übertragenen als auch im konkreten Sinne. An dieser Diskussion waren sowohl jüdische Ärzte und Rabbiner beteiligt, aber auch Nichtjuden, die in der jüdischen Gesundheitsvorsorge eine Bestätigung für neue wissenschaftliche Erkenntnisse sowie einen gewissen Vorbildcharakter erkannten. Das bedeutete, das mosaische Gesetz auf alltagsrelevante Praktiken zu reduzieren, die sich wissenschaftlich deuten und rechtfertigen ließen. Damit erreichte man gleichzeitig ein übergeordnetes Ziel, nämlich das Judentum von dem Deutungsmonopol des Rabbinertums zu befreien und es so zu modernisieren. In diesem Zusammenhang kam dem biblischen Moses die Funktion zu, den Urtyp des gesunden Juden zu verkörpern (Hart 2007, S. 18). Die nach ihm benannten mosaischen Gesetze wurden als richtungweisend für eine den menschlichen Körper gesunderhaltende Hygiene und Gesundheitsvorsorge gedeutet. So erklärte der jüdische Sozialhygieniker Alfred Nossig gegen Ende des 19. Jahrhunderts: „An die Spitze seiner hygienischen Gesetzgebung stellte also Moses das Gebot der Arbeit: und es ist das Verdienst der modernen Aerzte, welche die mosaische Hygiene untersucht, die Bedeutung dieses Gebotes beleuchtet zu haben“ (Nossig 1894, S. 32). Als besonderen Vorzug der mosaischen Hygiene hebt der Autor hervor, „dass sie den grossen Einfluss des moralischen Elementes auf die physische Gesundheit berücksichtigt und bewusst anwendet“ (Nossig 1894, S. 33) .Moses wird hier also als Vorbild für eine Gesundheitsgesetzgebung geschildert, die ganzheitliches Denken widerspiegelt, indem Leib und Seele als Einheit gedacht werden. Auch von christlicher Seite fehlte es im 19. Jahrhundert nicht an Stimmen, die meinten, dass man vom Judentum in dieser Hinsicht einiges lernen könne. So schrieb der amerikanische Arzt Edward H. Williams im Jahr 1882: 


\begin{abstract}
So sehr degeneriert, in moralischer Sicht, einzelne Abkömmlinge [der Israeliten, R. J.] heute auch sein mögen, so sind diese jedenfalls nicht in körperlicher Hinsicht degeneriert. Das ist eine ausreichende Antwort auf die oft wiederholte Behauptung, dass eine Zivilisation dazu tendiert, in physischer Hinsicht immer schwächer zu werden. Denn die Juden sind länger zivilisiert als jedes andere zivilisierte Volk. Können wir daher nicht daraus schließen, dass die Gebräuche eines Abraham oder Moses Anteil daran hatten, ein solches wunderbares Ergebnis zu erzielen, und dass es daher vielleicht von Nutzen wäre, dass diese von anderen Nationen nachgeahmt würden? (zitiert nach Hart 2007, S. 181).
\end{abstract}

Diesen Ratschlag beherzigen bis heute - wenn auch unwissentlich - viele gesundheitsbewusste Nichtjuden, die angesichts von Lebensmittelskandalen selbst Bio-Läden nicht mehr trauen, sondern koschere Lebensmittel einkaufen, weil in diesem Fall beispielsweise alle künstlichen Zusatzstoffe genauestens aufgelistet werden.

\title{
3 Die absolute Pflicht zur Gesunderhaltung
}

„Sorge für deine Gesundheit, bevor du krank wirst“, heißt es in der Bibel (Sirach 18:29). Nicht nur aus dieser apokryphen Schrift, auch aus der Thora selbst lässt sich nach rabbinischer Auslegung die Pflicht des Menschen zur Gesunderhaltung ableiten: „Hüte dich nur und bewahre deine Seele gut, dass du nicht vergisst, was deine Augen gesehen haben“ (5. Mose 4:9). Sogar konkrete Gefahren für Leib und Leben, die es zu vermeiden gilt, werden in der Bibel mit einem Warnhinweis versehen: „Wenn du ein neues Haus baust, so mache ein Geländer ringsum auf deinem Dache, damit du nicht Blutschuld auf dein Haus lädst, wenn jemand herabfällt“ (5. Mose 22:8). Dem Inhalt nach handelt es sich um eine frühe Unfallverhütungsvorschrift.

Die Sorge um ausreichende Ernährung findet sich ebenfalls in der Thora: „Wenn du vor einer Stadt lange Zeit liegen musst, gegen die du kämpfst, um sie zu erobern, so sollst du ihre Bäume nicht verderben und mit Äxten umhauen, denn du kannst davon essen; darum sollst du sie nicht fällen“ (5. Mose 20:19).

Da der Körper ein Werk Gottes ist, obliegt dieser nach jüdischer Auffassung der besonderen Sorge und Pflege. Alles, was der Körper benötigt (Essen, Trinken, Bewegung, Ruhe etc.), dient also dazu, dem Ewigen zu dienen, wie der Schulchan Aruch, die bekannteste jüdische Gesetzeskompilation, an einzelnen Beispielen aus dem alltäglichen Leben verdeutlicht (Kizzur Schulchan Aruch 1988, 31:7, S. 175 ff.). Diese wiederum beziehen sich größtenteils auf die Gesundheitslehre des Maimonides, in der es heißt: „Der Mensch muß sein Herz und alle seine Handlungen lediglich dahin bestimmen, daß er Gott, gelobt sei er, erkenne; sein Sitzen, 
Stehen, Reden, Alles sei darauf gerichtet“ (Maimonides 1846, S. 49). Und der berühmte Arzt und Philosoph fügt noch hinzu:

Weil nun Erhaltung der körperlichen Gesundheit und Stärke für ein Wandeln auf Gottes Wegen betrachtet wird, indem es unmöglich ist bei krankem Leibe etwas von der Erkenntniß Gottes zu begreifen und zu verstehen, darum muß sich der Mensch von dem Körper nachteiligen Dingen fern halten, wohl aber solche, die ihn stärken und erkräftigen beobachten.

Nicht die Gesunderhaltung an sich, sondern Gott zu dienen, sein Werk zu heiligen, steht hinter diesen Ratschlägen, die weitgehend der antiken Diätetik folgen. Maimonides verdeutlicht das mit Beispielen aus dem Alltag:

Wer nur in der Absicht nach den Gesundheitsregeln lebt, damit Körper und Glieder unversehrt bleiben, und damit er Kinder habe, die seine Arbeit verrichten und sich für seine Bedürfnisse abmühen, der hat keinen guten Weg eingeschlagen; er habe vielmehr die Absicht, daß sein Körper nur deshalb unversehrt und stark sei, damit seine Seele desto bereitwilliger werde, Gott zu erkennen, denn bei Hunger, Krankheit und Schmerz an irgend einem Gliede, ist es unmöglich Weisheitslehren zu erfassen und zu begreifen; er habe die Absicht, daß er einen Sohn erhalte, der vielleicht durch seine Weisheit und Größe einst eine Zierde in Israel werde. Wer auf diesem Wege sein Leben lang wandelt, der dienet Gott immerfort, selbst zu der Zeit, wann er sein Geschäft treibt oder seine eheliche Pflicht erfüllt; hat er doch bei Allem den Gedanken, nur darum seine Bedürfnisse zu erschwingen, daß sein Körper geneigt sei Gott zu dienen. Auch wenn er schläft und es nur in der Absicht thut, daß Seele und Körper sich ausruhen, damit er nicht krank und durch Krankheit verhindert werde Gott zu dienen, so ist auch dieser Schlaf ein Dienst Gottes, gelobt sei er. Dahin zielet das Gebot unserer Weisen: Alle deine Handlungen thue im Hinblick auf den himmlischen Namen; das bezeichnet auch Schelomoh in seiner Weisheit mit den Worten: „Auf all deinen Wegen merk’ auf ihn und er wird deine Pfade ebnen“ (Maimonides 1846, S. 50).

Besonders eindrücklich vermittelt außerdem ein Midrasch, eine rabbinische Schriftauslegung, die Pflicht eines jeden Juden zur Gesunderhaltung. Als der berühmte jüdische Gelehrte Hillel der Ältere (gest. um 10 n.Chr.) einmal von seinen Schülern nach einer Lehrstunde auf seinem Weg begleitet wurde, erkundigten sich diese, wohin er gehe. Als er zur Antwort gab, er wolle eine Pflicht erfüllen, kam die Nachfrage, welche diese denn sei. Daraufhin hörten seine Schüler zu ihrem Erstaunen, dass er vorhabe, ein Bad zu nehmen. Als sie wissen wollten, wieso das eine mizwa, also ein gutes Werk, sei, erklärte er ihnen: „Wie die Königsbilder im Theater und Circus von dem, dessen Obhut sie übergeben sind, reingehalten und abgespült werden müssen, so ist auch das Baden des Körpers eine Pflicht für den Menschen, der im Bilde des allmächtigen Königs geschaffen wurde“ (Leviticus Rabba 34,3 nach der Übersetzung von Bacher 1884, S. 10). Auch der Talmud betont beispielsweise, dass selbst Anweisungen, wie man am besten den Stuhlgang gestaltet, keineswegs als profan gelten dürfen, sondern so wichtig 
für die Gesunderhaltung des menschlichen Lebens sind, dass sie von kundigen Rabbinern weitergegeben werden. Als Rabbi Hona nämlich einmal seinen Sohn Rabba tadelte, warum er nicht Unterricht bei Rabbi Hisda nehme, rechtfertigte jener sich damit, dass dieser nur „weltliche Dinge“ lehre, unter anderem wie man Hämorrhoiden vermeiden kann. Darauf bekam er vom Vater die klare Antwort: „Er befasst sich mit der Gesundheitskunde der Menschen und du sagst, es seien weltliche Dinge! Erst recht sollst du zu ihm gehen“ (Der Babylonische Talmud 1967, 82a). Kein Kirchenvater hätte es gewagt, die menschliche Verdauung so konkret zu schildern und dazu auch noch Ratschläge zu erteilen.

In der Thora und vor allem im Talmud findet sich eine Vielzahl von Vorschriften, die belegen, wie sehr das Judentum die griechisch-römische Lehre von der Diätetik verinnerlicht, erweitert und an religiöse Bestimmungen angepasst hat. Dazu zählen die sex res non naturales (die sechs nicht natürlichen Dinge), auf die man nach der Lehre eines Hippokrates und Galen zu achten habe: Licht und Luft (aer), Speise und Trank (cibus et potus), Arbeit und Ruhe (motus et quies), Schlaf und Wachen (somnus et vigilia), Absonderungen und Ausscheidungen (secreta et excreta) sowie Anregung des Gemüts (affectus animi). Entsprechend reicht das diätetische Spektrum, das man im Talmud und anderen rabbinischen Texten vorfindet, von der Durchführung des bis ins 19. Jahrhundert üblichen Aderlasses bis hin zu detaillierten Verdauungsratschlägen (Grunwald 1912; Preuss 1992 [1911]). So bekommt man in den unterschiedlichsten Kontexten religiöser Praxis durchaus wertvolle Tipps zu Leibesübungen, zum Fasten, Händewaschen, Baden, Schlaf bis hin zur sexuellen Aktivität.Vieles von dem, was in diesen Texten an gesundheitsfördernden Maßnahmen empfohlen wird, ist auch heute noch nicht überholt. Man denke etwa an Ratschläge wie den, sich vor Völlerei zu hüten (,zahlreicher seien die Toten des Stuhles, als die vor Hunger geschwollenen“, Der Babylonische Talmud 1967, Schabbat 33a) oder sich nach dem Essen zu bewegen (,Wenn jemand gegessen hat und nicht vier Ellen gegangen ist, so bleibt die Speise unverdaut“, Der Babylonische Talmud 1967, Schabbat 41a). Dagegen sind die Empfehlungen zum Aderlass heute obsolet, da dieser in der Schulmedizin so gut wie keine Verwendung mehr findet; denn die Krankheitslehre hat sich verändert, die antike Humoralpathologie spielt inzwischen keine Rolle mehr. Auch die Empfehlung des Maimonides, Schaffelle statt Fuchs- oder Wieselfelle als Kleidung zu tragen, ist heute nicht mehr zeitgemäß. Es besteht daher in der jüngeren halachischen Literatur weitgehend Konsens darüber, dass die Gesundheitsfürsorge, wie sie vor allem im Talmud beschrieben wird, zeitbedingt war und man sich deshalb an heutigen Maßstäben und Erkenntnissen unter Einhaltung der Religionsgesetze orientieren sollte (Steinberg 2003, S. 834).

Jüngste Beispiel ist die COVID-19-Pandemie, die im Frühjahr 2020 in jüdischen Gemeinden das traditionelle Begehen des Auszugs aus Ägypten und die 
Befreiung aus der Sklaverei nicht nur in Deutschland erheblich erschwerte (Jütte 2020). Denn Abweichungen von den strengen Koscher-Vorschriften, die für dieses Fest gelten, bedürfen einer besonderen Rechtfertigung, die von der Halacha, dem jüdischen Religionsgesetz, vorgegeben sind. Dieses regelt bis ins kleinste Detail, wie Juden das Pessach-Fest feiern sollen. Abweichungen sind nur in einer Ausnahmesituation erlaubt. Eine solche ist immer dann gegeben, wenn es um die Rettung von Menschenleben geht. Dieses oberste Gebot im Judentum wird als Pikuach nefesch (wörtlich: Rettung der Seele) bezeichnet. So heißt es beispielsweise in der Mischna, der ältesten Schicht des Talmuds, im Traktat über den Versöhnungstag (Jom Kippur): „Wer Halsschmerzen hat, dem darf man auch am Sabbat Medizin geben, weil er möglicherweise in Lebensgefahr ist, und jede Lebensgefahr bricht [= verdrängt] den Sabbat“ (Mischna 2005, Joma 8.6.). Ausnahmen von dieser generellen Regel gelten nicht im Falle von Götzendienst, Unzucht und Mord. Diese Verbote dürfen also keinesfalls mit einer solchen Begründung übertreten werden.

Bestimmte Ausnahmen lassen sich auch mit dem talmudischen Prinzip Dina de-malchuta dina („Das Gesetz des Landes ist Gesetz“) legitimieren. Für Juden, die in der Diaspora leben, bedeutet das, dass sie grundsätzlich verpflichtet sind, die Gesetze des Landes, in dem sie leben, zu respektieren und zu befolgen. Das kann dazu führen, dass die Landesgesetze in bestimmten Fällen sogar vorrangig vor den Rechtsgrundsätzen der Halacha sein können. Allerdings lautet die überwiegende Lehrmeinung, dass dies nur für die sogenannten Mamona (Fragen des Zivil-, Steuer und Finanzrechts) und nicht für den religiösen Bereich gelte.

Bei der Vorbereitung auf Pessach 2020 erwies es sich für religiöse Juden als schwierig, die strengen Speisevorschriften für diese besonderen Festtage zu befolgen. Dazu zählt z. B. das Verbot, Chamez (gesäuertes Backwerk) im Besitz bzw. im Haus zu haben. Hinzukamen aufgrund von Lieferengpässen und Grenzsperren Probleme beim rechtzeitigen Erwerb von Nahrungsmitteln, die speziell für den Verzehr für Pessach durch ein entsprechendes Kaschrut-Zertifikat als erlaubt bezeichnet werden. So gestattete beispielsweise die Orthodoxe Rabbinerkonferenz den deutschen jüdischen Gemeinden, ausnahmsweise solche Lebensmittel zu verzehren, die nur den regulären Hechscher (Koscher-Stempel) aufweisen. Unter den Nahrungsmitteln, die in der herrschenden Krise auch ohne Koscher-le-Pessach-Zertifikat gekauft werden durften, sind unter anderem frisches Obst und Gemüse (mit Ausnahme von bestimmten Hülsenfrüchten, sogenannten Kitnijot, diese allerdings nur nach aschkenasischer Tradition).

Die Empfehlungen religiöser Autoritäten für den Einkauf koscherer Lebensmittel für das Pessachfest 2020 sind ein eindrücklicher Beleg für die religiöse Sorgfaltspflicht in Hinblick auf die Einhaltung der halachischen Vorschriften für den Pessach-Einkauf in Corona-Zeiten. Die in dieser Situation geltenden Aus- 
nahmeregelungen nehmen soweit es geht Rücksicht auf die alltäglichen Bedürfnisse der modernen Lebenswelt. Sie machen aber gleichzeitig klar, worauf ein Verzicht auch in Krisenzeiten undenkbar scheint.

\section{Literatur}

Bacher, Wilhelm (1884): Die Agada der Tannaiten: Von Hillel bis Akiba. Von 30 vor bis 135 nach d. g. Z. Straßburg: Karl J. Trübner.

Baneth, Berthold (1912): „Das jüdische Ritualgesetz in hygienischer Beleuchtung“. In: Max Grunwald (Hrsg.): Die Hygiene der Juden. Im Anschluß an die Internationale Hygiene-Ausstellung Dresden 1911. Dresden: Verlag der Historischen Abteilung der Internationalen Hygiene-Ausstellung, S. 43-102.

Buxtorf, Johann (1643): Synagoga Judaica. Das ist Juden-Schul [...]. Basel: Ludwig König.

Der Babylonische Talmud (1967). Neu übertr. durch Lazarus Goldschmidt (2. Aufl.). Berlin: Jüdischer Verlag.

Epstein, Ephraim M. (1874): „Have the Jews any Immunity from Certain Diseases?“. In: The Medical and Surgical Reporter 30, S. 342-344.

Gilman, Sander L. (1995): Picturing Health and Illness. Images of Identity and Difference. Baltimore \& London: Johns Hopkins University Press.

Grunwald, Max (Hrsg.) (1912): Die Hygiene der Juden. Im Anschluß an die Internationale Hygiene-Ausstellung Dresden 1911. Dresden: Verlag der Historischen Abteilung der Internationalen Hygiene-Ausstellung.

Hart, Mitchell Bryan (2007): The Healthy Jew: The Symbiosis of Judaism and Modern Medicine. Cambridge: Cambridge University Press.

Jütte, Robert (2016): Leib und Leben im Judentum. Berlin: Jüdischer Verlag.

Jütte, Robert (2020): „Vorbereitungen zu Pessach in Zeiten von COVID-19“. Hamburger Schlüsseldokumente zur deutsch-jüdischen Geschichte. https://juedische-geschichteonline.net/beitrag/jgo:article-262, besucht am 15.4.2020.

Kaiser, Otto (2002): „Krankheit und Heilung nach dem Alten Testament“. In: Medizin, Gesellschaft und Geschichte 20, S. 9-43.

Kizzur Schulchan Aruch (1988). Ins Deutsche übertragen von Rabbiner Dr. Selig Bamberger (Neue, verbesserte Aufl.). Basel: Victor Goldschmidt.

Krauskopf, Samuel (1889): Sanitary Science. A Sunday Lecture. Philadelphia, PA: S. W. Goodman.

Leder, Christoph Maria (2007): Die Grenzgänge des Marcus Herz: Beruf, Haltung und Identität eines jüdischen Arztes gegen Ende des 18. Jahrhunderts. Münster: Waxmann.

Levin, Jeff/Prince, Michele F. (2010): ,Judaism and Health: Reflections on an Emerging Scholarly Field“. In: Journal of Religion and Health 50(4), S. 765-777.

Maimonides, Moses (1846): Jad hachasakah; oder, Mischna Thorah: Erstes Buch (Elias Soloweiczyk, Hrsg.). Königsberg: E. J. Dalkowski.

Mischna (2005). Ins Deutsche übertragen, mit einer Einleitung und Anmerkungen von Dietrich Correns. Wiesbaden: Marix.

Nossig, Alfred (1894): Die Sozialhygiene der Juden und des altorientalischen Völkerkreises. Stuttgart: Deutsche Verlags-Anstalt. 
Preuss, Julius (1992 [1911]): Biblisch-talmudische Medizin. Beiträge zur Geschichte der Heilkunde und der Kultur überhaupt. Wiesbaden: Fourier.

Schuster, Josef (1980): „Zur Sterblichkeit jüdischer und nicht-jüdischer Säuglinge“ [Dissertation, Universität Würzburg]. Würzburg.

Steinberg, Avraham (2003): Encyclopedia of Jewish medical ethics: a compilation of Jewish medical law on all topics of medical interest, from the most ancient sources to the most current deliberations and decisions, with a concise medical and historical background, and a comprehensive comparative analysis of relevant general ethical approaches (Fred Rosner, Übers.). Jerusalem: Feldheim Publishers.

Susser, Mervyn W./Watson, William (1972): Sociology in Medicine (2. Aufl.). New York, NY: Oxford University Press.

Tacitus, Publius Cornelius (1984): Historien. Lateinisch und deutsch. Übersetzt, erläutert und herausgegeben von Helmuth Vrteska. Stuttgart: Reclam.

Wiesemann, Falk (1993): „Hygiene der Juden auf der Düsseldorfer GESOLEl 1926. Jüdische Kulturleistungen in der Weimarer Republik“. In: Geschichte im Westen 8, S. 24-37.

Wolff, Eberhard (2014): Medizin und Ärzte im deutschen Judentum der Reformära: die Architektur einer modernen jüdischen Identität. Göttingen: Vandenhoeck \& Ruprecht. 